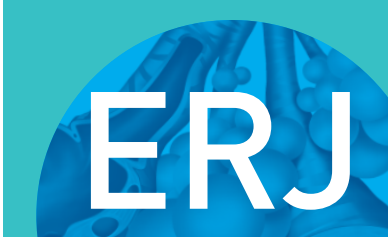

open research

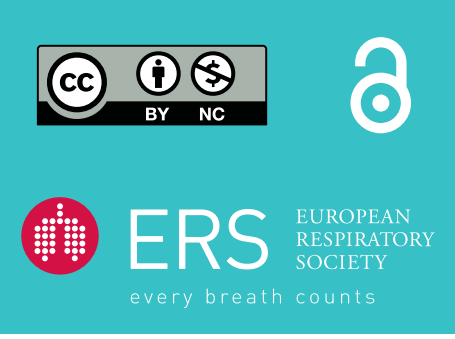

\section{Rapid decline of seasonal influenza during the outbreak of COVID-19}

\author{
To the Editor:
}

Seasonal influenza is a leading cause of disease worldwide, resulting in as many as 650000 deaths on a yearly basis [1]. Vaccination is the cornerstone of influenza prevention, but its efficacy is limited by the accuracy in predicting circulating strains. Epidemiological methods such as social distancing, the use of protective face masks and frequent hand washing are not routinely practiced in influenza prevention. In contrast, these methods have been widely applied during the COVID-19 outbreak. In this study, we investigated how this year's influenza season has been impacted during the COVID-19 pandemic.

Influenza incidence was obtained from the World Health Organization (WHO) website https://www.who. int/influenza/gisrs_laboratory/flunet/en/. COVID-19 cases were also sourced from the WHO https:// experience.arcgis.com/experience/685d0ace521648f8a5beeeee1b9125cd. Data were analysed from the first three countries with major COVID-19 outbreaks of over 75000 cases: China, Italy and the USA. To account for yearly variation in the timing of newly reported influenza cases, we assigned the peak in each country (the week in which the most cases of influenza were reported) as week 0 (figure 1). After aligning the peaks of different influenza seasons, we calculated the mean number of cases for each week flanking the peak over the 2015/2016, 2016/2017, 2017/2018 and 2018/2019 seasons. Using linear regression models, we quantified the rate of decline for each country in newly reported cases of influenza from the influenza peak to weeks 4 and 7 for the 2019/2020 season compared with the mean 2015-2019 experience. The $\mathrm{p}$-values for the interaction term for season and rates of decline by week were calculated and $\mathrm{p}<0.05$ was considered significant.

In China and Italy, there was a steeper decline in new influenza cases reported during the first 4 weeks following the influenza peak this season when compared with prior years (2015-2019) ( $\mathrm{p}=0.004$ and $\mathrm{p}=0.001$, respectively). This rapid decline was temporally associated with the substantial outbreak of COVID-19 (exceeding 10000 cases) during the first 4 weeks following the influenza peak in these countries.

In contrast, in comparing this season to prior years (2015-2019), there was not a significantly steeper decline in new influenza cases reported during the first 4 weeks following the influenza peak in the USA $(\mathrm{p}=0.60)$. Notably in the USA, the substantial outbreak of COVID-19 cases lagged behind the other two countries, only exceeding 10000 cases by 7 weeks following the influenza peak. Quantifying the decline in new influenza cases reported during the first 7 weeks following the influenza peak this season in the USA, there was a steeper decline in influenza cases this season when compared with prior years $(\mathrm{p}=0.003)$.

This analysis demonstrates that there was an unusually rapid rate of decline of new influenza cases reported in three countries highly impacted by the COVID-19 pandemic. In China and Italy, COVID-19 cases rose substantially within 4 weeks following the influenza peak, correlating with a significantly steeper decline in newly reported influenza cases compared to prior years. In contrast, the USA did not have a significantly steeper decline in influenza cases in the first 4 weeks following the peak. However, it is notable that the outbreak of COVID-19 hit the USA later than China and Italy. This delay relative to the influenza peak, potentially combined with less aggressive control measures against community spread, may have contributed to the lag in the decline of influenza cases until 7 weeks after the peak.

The precise mechanisms explaining the rapid decline in influenza cases this year may be multifactorial, but we hypothesise that the implementation of public health measures such as social distancing, the use of

@ERSpublications

The implementation of public health measures during the \#COVID19 pandemic may also help to reduce transmission of respiratory illnesses such as influenza https://bit.ly/2BmysRJ

Cite this article as: Young G, Peng X, Rebaza A, et al. Rapid decline of seasonal influenza during the outbreak of COVID-19. ERJ Open Res 2020; 6: 00296-2020 [https://doi.org/10.1183/ 23120541.00296-2020].

Copyright $\odot$ ERS 2020. This article is open access and distributed under the terms of the Creative Commons Attribution NonCommercial Licence 4.0. 


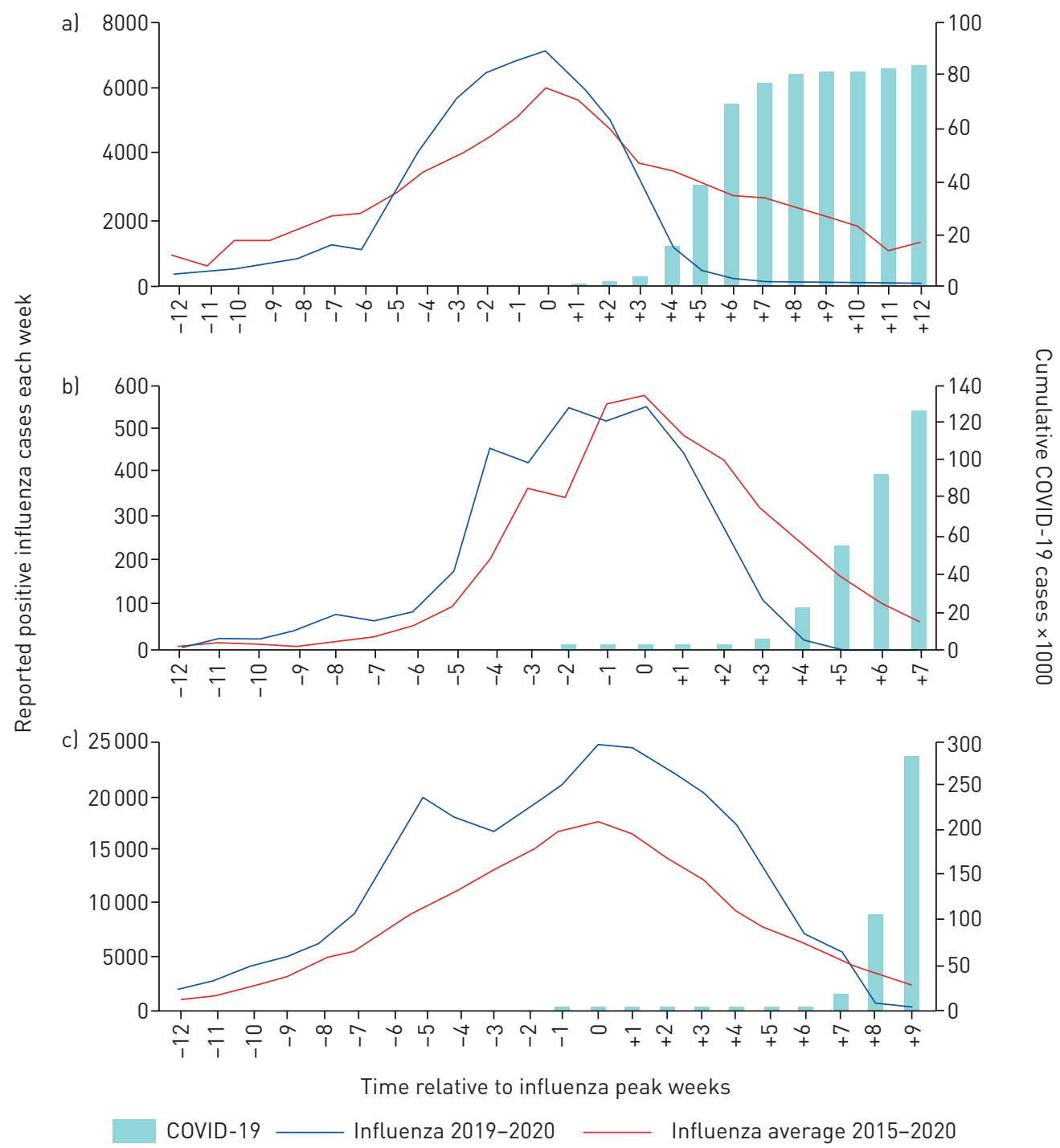

FIGURE 1 Newly reported influenza cases in a) China, b) Italy and c) the USA in 2019/2020 (blue) compared to the average for 2015-2019 influenza seasons (red). The $x$-axis shows weeks leading up to and following the peak at week 0 . Newly reported influenza cases are shown on the left $y$-axis. COVID-19 cases (green) are shown on the right $y$-axis.

protective face masks and frequent hand washing following the outbreak of COVID-19 might have contributed to our observation. It is possible that fewer influenza tests were performed this year due to the focus on COVID-19. However, in China, influenza A and B were typically tested in suspected COVID-19 cases with flu-like symptoms [2]. In addition, in the USA, the WHO reported high levels of influenza testing after the outbreak of COVID-19, with a similar number of influenza tests at the peak and 7 weeks after the peak [3]. We also cannot exclude the influence of viral interference (an immune response to one viral infection prevents a second infection) in limiting influenza spread, although co-infection with influenza and SARS-CoV-2 has been reported [4].

China, Italy and the USA were prominent in the early headlines surrounding COVID-19, and represent three continents that have been heavily impacted by the pandemic. Extending this analysis to other countries was limited by delays or underreporting of new influenza cases in many countries on the WHO website. However, subsequent investigations should explore the findings of this study on a broader scale, perhaps including dozens of countries that experienced COVID-19 outbreaks. A larger analysis could address the impact of social distancing more directly, interrogating whether different countries' policies during the COVID-19 pandemic correlates with tangible differences in the decline of newly reported influenza cases this year compared to prior seasons. 
Seasonal influenza has been a major clinical problem for many decades. However, the public health efforts to prevent influenza spread by limiting human-to-human transmission remain minimal. The current strategy to limit the COVID-19 pandemic has emphasised behavioural measures, such as the use of face masks and effective hand washing. The adoption of these measures to contain the spread of seasonal influenza should be investigated. Furthermore, there is concern about the potential impact of a second wave of COVID-19 during the upcoming influenza season in the upcoming autumn. The implementation of public health measures in the early fall based on the first indication of an outbreak of either influenza or COVID-19 may help to reduce disease transmission of both respiratory illnesses.

\section{Grant Young ${ }^{1,4}$, Xiaohua Peng ${ }^{1,4}$, Andre Rebaza ${ }^{2}$, Santos Bermejo ${ }^{1}$, Chang De ${ }^{3}$, Lokesh Sharma ${ }^{1,5}$ and Charles S. Dela Cruz ${ }^{1,5}$ \\ ${ }^{1}$ Yale School of Medicine, Dept of Internal Medicine, Section of Pulmonary, Critical Care, and Sleep Medicine, New Haven, CT, USA. ${ }^{2}$ Yale School of Medicine, Dept of Internal Medicine, Section of Pediatrics, New Haven, CT, USA. ${ }^{3}$ College of Respiratory and Critical Care Medicine, Chinese PLA General Hospital, Beijing, China. ${ }^{4}$ Joint first authors. ${ }^{5}$ Joint senior authors.}

Correspondence: Lokesh Sharma, 300 Cedar Street, TAC S-440, New Haven, CT 06520, USA. E-mail: lokeshkumar.sharma@yale.edu; Charles S. Dela Cruz, 300 Cedar Street, TAC S-440, New Haven, CT 06525, USA. E-mail: charles.delacruz@yale.edu

Received: 22 May 2020 | Accepted after revision: 29 May 2020

Published online 17 August, 2020; republished 21 August, 2020 with amendments to an author family name

Conflict of interest: None declared.

\section{References}

1 World Health Organization. Influenza (Seasonal). www.who.int/en/news-room/fact-sheets/detail/influenza-(seasonal). Date last accessed: 27 April 2020.

2 Huang C, Wang Y, Li X, et al. Clinical features of patients infected with 2019 novel coronavirus in Wuhan, China. Lancet 2020; 395: 497-506.

3 World Health Organization. Influenza. www.who.int/influenza/gisrs_laboratory/flunet/en/. Date last accessed: 27 April 2020.

4 Wu X, Cai Y, Huang X, et al. Co-infection with SARS-CoV-2 and influenza A virus in patient with pneumonia, China. Emerging Infect Dis 2020; 26: 1324-1326. 